\title{
Erratum to: Effect of taurine supplementation on hepatic metabolism and alleviation of cadmium toxicity and bioaccumulation in a marine teleost, red sea bream, Pagrus major
}

\author{
Takeshi Hano • Katsutoshi Ito • Kumiko Kono • \\ Mana Ito • Nobuyuki Ohkubo • Kazuhiko Mochida \\ Published online: 11 January 2017 \\ (C) Springer Science+Business Media Dordrecht 2017
}

Erratum to: Fish Physiology and Biochemistry

DOI: $10.1007 / \mathrm{s} 10695-016-0274-7$

The published online version of this article contained some incorrect numerical values under the last column of Table 4 . In the published online version, the following numerical values were listed: $1.4 \pm 0.2,1.3 \pm 0.2$, and $1.2 \pm 0.1$.

However, the correct numerical values should be: $29.1 \pm 10.8 x y, 21.9 \pm 7.1 z$, and $17.4 \pm 4.4 x z$. The corrected version of Table 4 is shown below.

The online version of the original article can be found at http://dx. doi.org/10.1007/s10695-016-0274-7

T. Hano $(\bowtie) \cdot$ K. Ito $\cdot$ K. Kono $\cdot$ M. Ito $\cdot$ N. Ohkubo $\cdot$ K. Mochida

Fisheries Research and Education Agency, National Research Institute of Fisheries and Environment of Inland Sea, 2-17-5,

Maruishi, Hatsukaichi, Hiroshima 739-0452, Japan

e-mail: hntks@affrc.go.jp

URL: http://feis.fra.affrc.go.jp/ 
Table 4 Cd concentrations $(\mu \mathrm{g} / \mathrm{g})$ in the liver and muscle

\begin{tabular}{|c|c|c|c|c|c|c|c|c|c|c|c|c|c|c|c|c|}
\hline \multirow[b]{2}{*}{ Liver $^{4}$} & \multirow[t]{2}{*}{$\begin{array}{l}0 d^{1} \\
(\text { day } 40)\end{array}$} & \multicolumn{3}{|c|}{$\begin{array}{l}7 d^{2,3} \\
\text { (day 47) }\end{array}$} & \multicolumn{3}{|c|}{$\begin{array}{l}14 d^{2,3} \\
\text { (day 54) }\end{array}$} & \multicolumn{3}{|c|}{$\begin{array}{l}28 d^{2} \\
\text { (day 68) }\end{array}$} & \multicolumn{3}{|c|}{$\begin{array}{l}35 \mathrm{~d}(\text { eli } 7 \mathrm{~d})^{2,3} \\
(\text { day } 75)\end{array}$} & \multicolumn{3}{|c|}{$\begin{array}{l}\text { 49d (eli 21d })^{2} \\
(\text { day } 89)\end{array}$} \\
\hline & & & & & & & & & & & & & & & & \\
\hline TAU0 \% & B.D.L & 17.1 & \pm & $10.3^{\mathrm{ax}}$ & 22.8 & \pm & $6.9^{\mathrm{ax}}$ & 33.5 & \pm & $12.6^{x y}$ & 49.4 & \pm & $19.7^{\text {ay }}$ & 29.1 & \pm & $10.8^{x y}$ \\
\hline TAU $0.5 \%$ & B.D.L & 5.6 & \pm & $2.4^{b x}$ & 19.6 & \pm & $4.0^{\mathrm{abxz}}$ & 38.3 & \pm & $13.6^{y}$ & 29.5 & \pm & $6.2^{\text {abyz }}$ & 21.9 & \pm & $7.1^{\mathrm{z}}$ \\
\hline TAU $5.0 \%$ & B.D.L & 4.9 & \pm & $0.5^{\mathrm{bw}}$ & 14.4 & \pm & $1.9^{\mathrm{bx}}$ & 27.9 & \pm & $4.4^{\mathrm{y}}$ & 19.6 & \pm & $3.7^{\text {byz }}$ & 17.4 & \pm & $4.4^{x z}$ \\
\hline \multicolumn{17}{|l|}{ Muscle } \\
\hline TAU0 \% & B.D.L & 0.022 & \pm & $0.004^{\mathrm{a}}$ & 0.035 & \pm & 0.012 & 0.017 & \pm & 0.006 & 0.026 & \pm & 0.015 & 0.040 & \pm & 0.029 \\
\hline TAU $0.5 \%$ & B.D.L & 0.014 & \pm & $0.012^{\mathrm{a}, \mathrm{b}}$ & 0.021 & \pm & 0.010 & 0.033 & \pm & 0.015 & 0.026 & \pm & 0.016 & 0.021 & \pm & 0.008 \\
\hline TAU $5.0 \%$ & B.D.L & 0.006 & \pm & $0.004^{\mathrm{b}}$ & 0.023 & \pm & 0.008 & 0.036 & \pm & 0.021 & 0.016 & \pm & 0.007 & 0.028 & \pm & 0.025 \\
\hline
\end{tabular}

${ }^{1}$ B.D.L below detection limit

${ }^{2}$ Values are expressed as means \pm standard deviation

${ }^{3}$ For each tissue, different superscript letters $(\mathrm{a}, \mathrm{b}, \mathrm{c})$ in a column indicate significant differences in Cd concentration between diets $(p<0.05)$

${ }^{4}$ Different superscript letters $(\mathrm{w}, \mathrm{x}, \mathrm{y}, \mathrm{z})$ in a row indicate significant differences in Cd concentration between sample days $(p<0.05)$ 